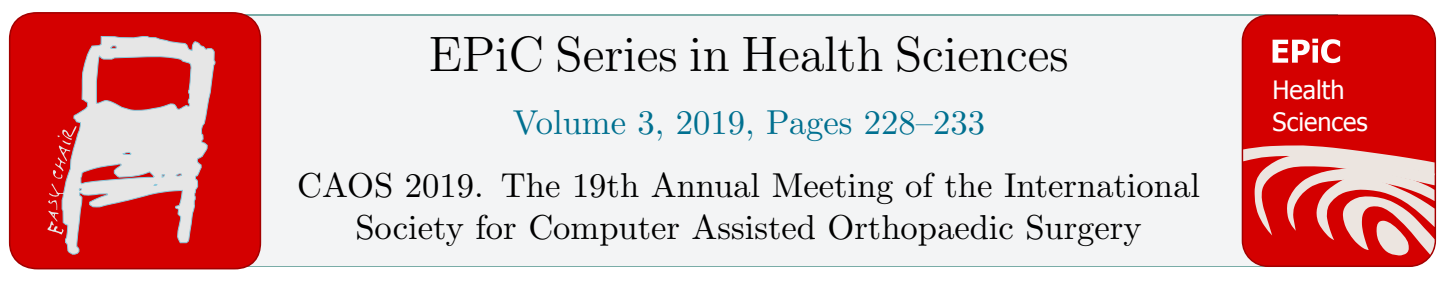

\title{
Spontaneous Healing of Brown-Sequard Syndrome Caused by Cervical Disc Herniation
}

\author{
Liu Xiaoyun, Hu Hongzhi, Shao Zhengwu, Xie Mao \\ Department of Orthopaedic Surgery, Wuhan Union Hospital, Tongji Medical \\ College, Huazhong University of Science and Technology \\ xylxyhzzh@126. com
}

\begin{abstract}
Brown-Sequard syndrome (BSS) is most commonly seen in patients with spinal trauma and extramedullary spinal neoplasm. Pure BSS caused by cervical disc herniation is extremely rare. Operative treatment is generally recommended those patients with BSS to improve neurological function. Here, we report a rare case of spontaneous healing of BSS caused by cervical disc herniation. Two years follow-up showed complete disappearance of symptoms without recurrence. To our knowledge, no similar cases have been reported before. Therefore, for appropriate patients, conservative treatment can be considered for a few months before deciding on surgical treatment.
\end{abstract}

Key Words: Brown-Sequard syndrome; Cervical disc herniation; Spontaneous; healing

\section{Introduction}

BSS is usually characterized by ipsilateral motor weakness, loss of proprioceptive and vibratory sensation, as well as loss of contralateral pain and temperature sensation ${ }^{1}$. BSS is most commonly seen in patients with spinal trauma and extramedullary spinal neoplasm ${ }^{2-4}$. However, cervical disc herniation is an uncommon cause of BSS and only few cases have been reported ${ }^{5-7}$. Early surgical decompression of the spinal cord is generally recommended to obtain an improvement in neurological function ${ }^{8}$. Here, we report a case of spontaneous healing of BSS caused by C5-6 herniated cervical disc lead to a severe spinal cord compression. To our knowledge, this is the first case to report such condition. In addition, the potential mechanisms have also been discussed. 


\section{Case Report}

A 45-year-old woman who was unable to conduct her routine activities presented with a 5-month history of gradual-onset neck pain and numbness and weakness of the right upper extremity accompanied with a 3-month history of weakness and paresthesias of left lower extremity. She denied any history of trauma to the head or neck. Her neurologic examinations revealed motor weakness of the right upper extremity and left lower extremity (Manual Muscle Test 3/5), in association with pain and temperature sensation loss on the left side below the T10 dermatome. Fortunately, there was no bladder or bowel dysfunction. On deep tendon reflex examination, the Hoffman sign was positive on the right side and the ankle clonus test was positive on the left side. All these findings were in accordance with a diagnosis of BSS caused by cervical disc herniation. The basic $\mathrm{x}$-rays of the cervical spine revealed no abnormality. However, magnetic resonance (MR) images showed extruded herniated nucleus pulposus (HNP) at the C5-6 level, almost occupying half of the vertebral canal volume (Fig.1).

Surgical treatment was suggested, but the patient refused. Then the patient was received conserve management in the form of intravenous $250 \mathrm{ml} 20 \%$ mannitol plus $10 \mathrm{mg}$ dexamethasone once a week for four weeks. In addition, non-steroidal anti-inflammatory drugs (celebrex $200 \mathrm{mg}$ orally once a day) and physiotherapy (a warm water bath once a day for at least 30 minutes at a time) were also recommended.

Incredibly, the patient's symptoms gradually relieved and followed up as an outpatient. About 2 months later, she showed a significant improvement in right-sided motor weakness. And the left-sided pain and temperature sensation gradually recovered 4 months later. At the 10-month follow-up examination revealed a complete recovery from motor deficits and full recovery of the left-sided pain and temperature sensation. No pathological reflection was induced. About 1 year later, she has since started to return to her normal life and the follow-up cervical MRI revealed almost complete regression of the extruded disc at the C5-6 level without any neural compression. Moreover, during 2 years of follow-up, the patient remained asymptomatic and had no recurrence and the MRI still confirmed almost complete regression of the extruded disc (Fig.2, Fig.3).

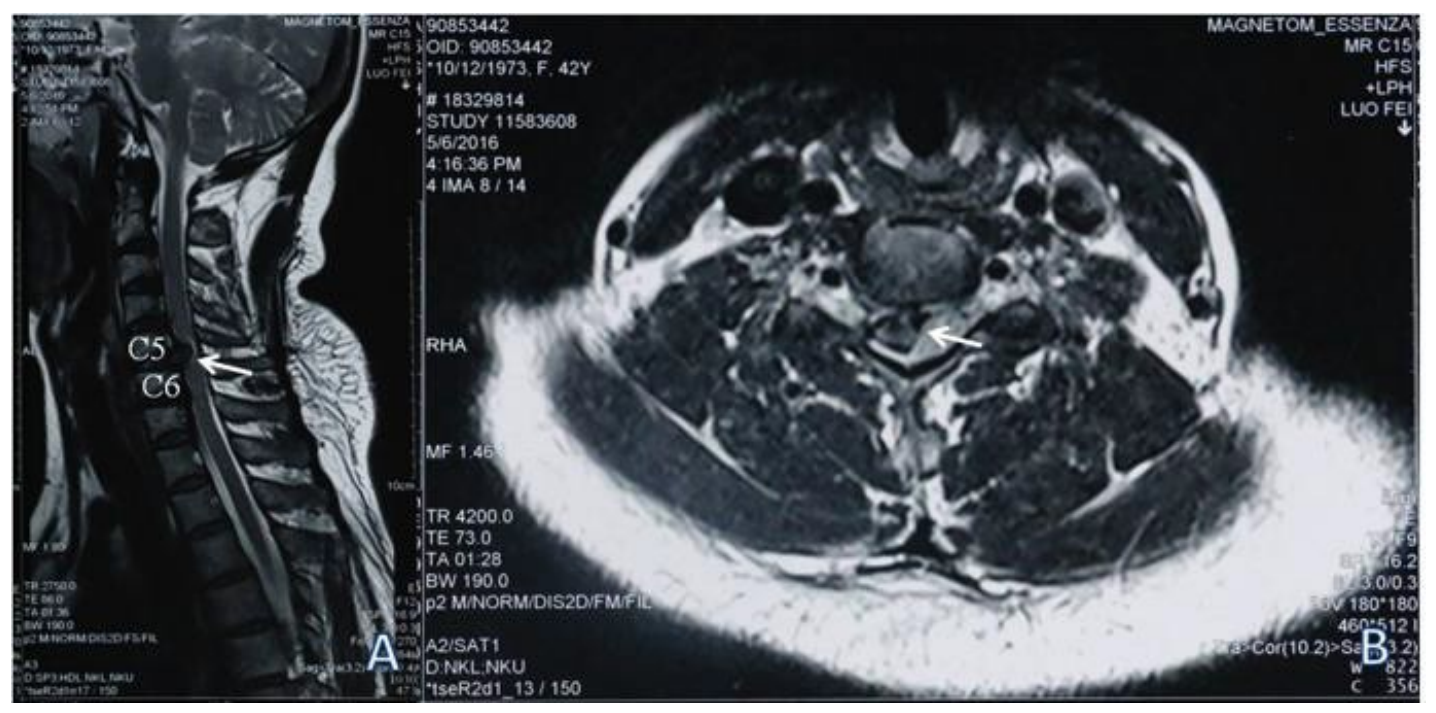

Fig.1: The initial MRI (May 6, 2016) study of the cervical spine (A, mid-sagittal; B, axial) revealed a large herniated disc (arrow) at the C5-6 level compressing the right C6 root 


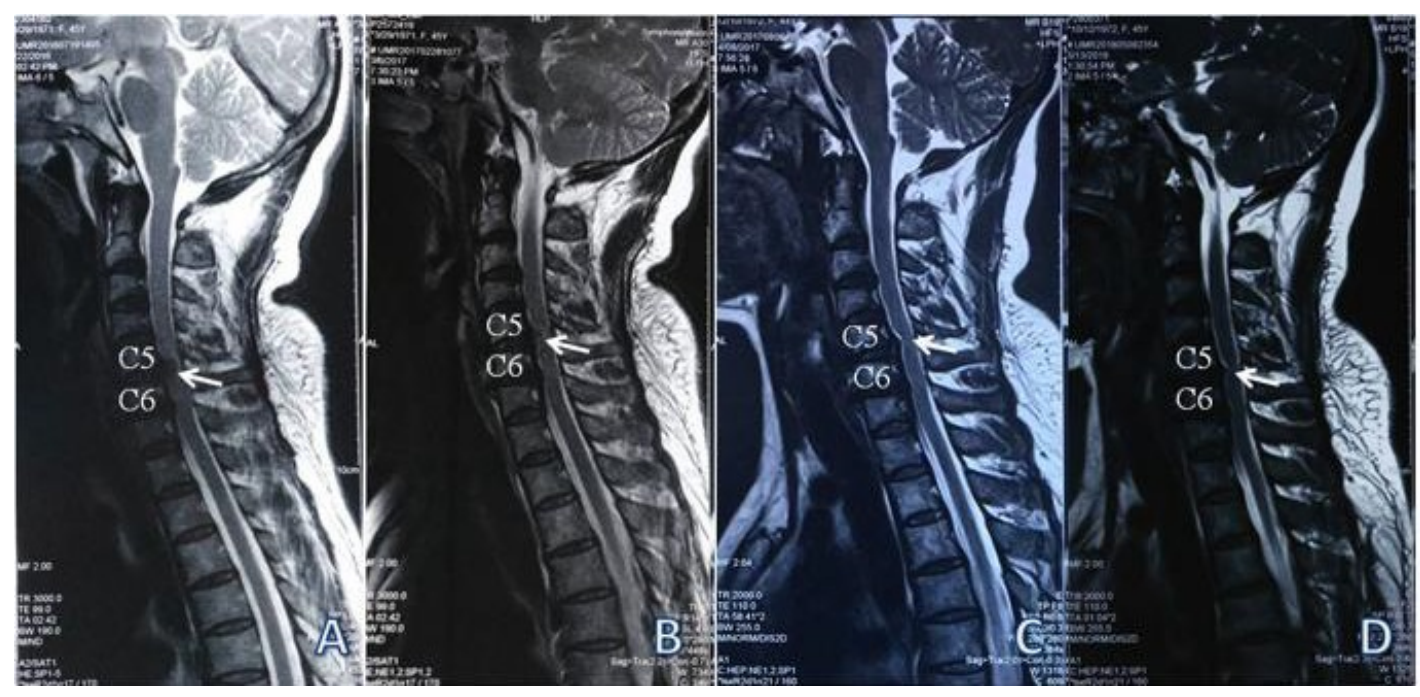

Fig.2: Follow-up sagittal MRI (A, July 22, 2016 ; B, March 6, 2017; C, August 14, 2017 ; D, May 13,2018) study of the cervical spine showed the gradual regression of the extrude disc (arrow) at the C5-6 level.

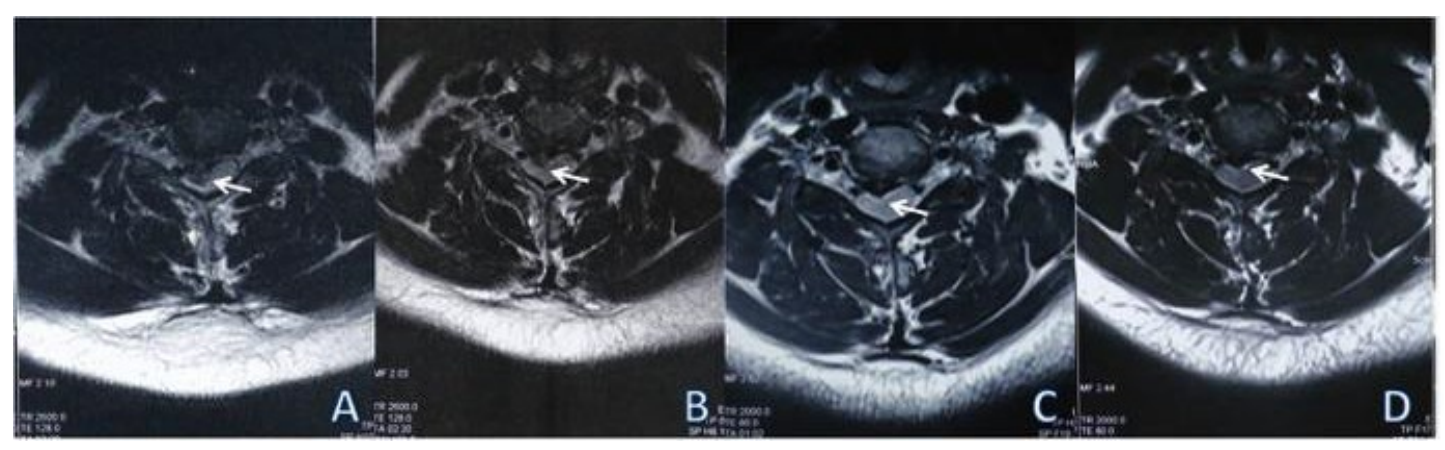

Fig.3: Follow-up axial MRI (A, July 22, 2016 ; B, March 6, 2017; C, August 14, 2017 ; D, May 13,2018) study of the cervical spine showed the gradual regression of the extrude disc (arrow) at the C5-6 level.

\section{Discussion}

Cervical disc herniation, which induces upper extremity pain and neurological deficits, appears a highly prevalent diseases that neurosurgeons and orthopaedic surgeons face every day. Cervical disc herniation is an uncommon cause of BSS, of which C5-C6 is the most frequently involved ${ }^{5,9}$. In present case, MRI showed a large central right-sided C5-C6 disc herniation, almost occupying half of the vertebral canal volume, severely compressing the spinal cord. As a consequence, the neural compression seen in our patient was primarily on the spinal cord itself rather than the nerve root. Many studies have shown that the sensory level of spinal cord was usually three segments lower than the lesion of BSS caused by cervical disc herniation ${ }^{10}$. In our case, the sensory level is at the level T10 dermatome on the left side while the compressive lesion is at C5-6 on the right side. 
Serial MRI can be used to demonstrate that spontaneous regression of disc herniation correlates with clinical improvement ${ }^{11}$. There is a direct correlation between the spontaneous disc regression and clinic improvement, which confirmed with MRI images ${ }^{12}$. In our case, about 10 months after receiving the conservative treatment mentioned above, a follow-up physical examination revealed a complete recovery from motor deficits and full recovery of the left-sided pain and temperature sensation and the cervical MRI showed almost half of the herniated disc spontaneously absorbed. Subsequently, one and two-year follow-up MRI revealed almost complete regression of the extruded disc at the C5-6 level without any neural compression.

Numerous studies have indicated that herniated cervical discs have the potential to spontaneously regress ${ }^{13-15}$. There are three popular mechanisms assumed in the literature explaining the process. The first mechanism is retraction theory, which presumes the herniated disc may retract back into the intervertebral disc space, but it only occurs when the herniated intervertebral disc has protruded through the annulus fibrosis without separating from $i t^{16,17}$. The second, dehydration theory, hold that disc regression is due to gradual dehydration and shrinkage ${ }^{18-20}$. The last mechanism, with the most extensive research and the farthest influence, involves inflammatory reaction with neovascularization ${ }^{16,20,21}$. In this view, the herniated disc, as a foreign body, induces an inflammatory reaction and neovascularization leading to enzymatic degradation and phagocytosis of cartilaginous tissue ${ }^{21-23}$. It appears that the vascular supply played a far more important role in resorption of the disc material ${ }^{21}$.

In order to obtain an improvement in neurological function, early surgical decompression of the spinal cord is generally recommended for patients with BSS caused by cervical disc herniation. However, decompression on the injured spinal cord during surgery may increase spinal cord damage ${ }^{24}$. Previous studies showed that treatment should be conservative in the initial course of the sequestrated type of disc herniation for at least 2 months before recommending surgical intervention unless severe neurologic deterioration occurs ${ }^{25}$. In our patient, with conservative treatment, the symptoms begin to ease about 2 months later, and complete relief after 10 months. But what should be taken into account seems a certain amount of the risk of the occurrence of neurological deterioration when select the conservative treatment. During the clinical course, what seems difficult is to predict which patient will profit from conservative treatment and which patient will require surgery. Therefore, a further study should be done to discover its real mechanism.

\section{Conclusion}

BSS caused by cervical disc herniation is extremely rare, therefore, detailed neurological examination and careful medical history collection are essential for early diagnosis. More and more studies have shown that cervical disc herniation have the potential to spontaneously regress through conservative treatment. In our opinion, for appropriate patients, conservative treatment can be considered for a few months before deciding on surgical treatment.

\section{Conflict of Interests}

The authors declare that they have no conflict of interests, financial or otherwise. 
Spontaneous Healing of Brown-Sequard Syndrome Caused by Cervical Disc Herniation X. Liu et al.

\section{References}

1. Kim JT, Bong HJ, Chung DS, Park YS. Cervical disc herniation producing acute brownsequard syndrome. Journal of Korean Neurosurgical Society 2009;45:312-4.

2. Aryan HE, Farin A, Nakaji P, Imbesi SG, Abshire BB. Intramedullary spinal cord metastasis of lung adenocarcinoma presenting as Brown-Sequard syndrome. Surgical neurology 2004;61:72-

6.

3. Block F. [Clinical picture of spinal tumors]. Radiologe 2006;46:1025-8.

4. Pouw $\mathrm{MH}$, van de Meent $\mathrm{H}$, van Middendorp JJ, et al. Relevance of the diagnosis traumatic cervical Brown-Sequard-plus syndrome: an analysis based on the neurological and functional recovery in a prospective cohort of 148 patients. Spinal Cord 2010;48:614-8.

5. Porto GB, Tan LA, Kasliwal MK, Traynelis VC. Progressive Brown-Sequard syndrome: A rare manifestation of cervical disc herniation. J Clin Neurosci 2016;29:196-8.

6. Yokoyama K, Kawanishi M, Yamada M, Kuroiwa T. Cervical disc herniation manifesting as a Brown-Sequard syndrome. Journal of neurosciences in rural practice 2012;3:182-3.

7. Choi KB, Lee CD, Chung DJ, Lee SH. Cervical disc herniation as a cause of brown-sequard syndrome. Journal of Korean Neurosurgical Society 2009;46:505-10.

8. Meng Y, Zhou L, Liu X, Wang H, Shi J, Guo Y. Brown-Sequard syndrome associated with Horner syndrome following cervical disc herniation. Spinal Cord Ser Cases 2016;2:16037.

9. Abouhashem S, Ammar M, Barakat M, Abdelhameed E. Management of Brown-Sequard syndrome in cervical disc diseases. Turk Neurosurg 2013;23:470-5.

10. Kobayashi N, Asamoto S, Doi H, Sugiyama H. Brown-Sequard syndrome produced by cervical disc herniation: report of two cases and review of the literature. The spine journal : official journal of the North American Spine Society 2003;3:530-3.

11. Kim SG, Yang JC, Kim TW, Park KH. Spontaneous regression of extruded lumbar disC herniation: three cases report. Korean J Spine 2013;10:78-81.

12. Citisli V, Ibrahimoglu M. Spontaneous remission of a big subligamentous extruded disc herniation: case report and review of the literature. Korean Journal of Spine 2015;12:19-21.

13. Han SR, Choi CY. Spontaneous regression of cervical disc herniation: a case report. Korean J Spine 2014;11:235-7.

14. Gurkanlar D, Yucel E, Er U, Keskil S. Spontaneous regression of cervical disc herniations. Minim Invasive Neurosurg 2006;49:179-83.

15. Song JH, Park HK, Shin KM. Spontaneous regression of a herniated cervical disc in a patient with myelopathy. Case report. J Neurosurg 1999;90:138-40.

16. Guinto FC, Jr., Hashim H, Stumer M. CT demonstration of disk regression after conservative therapy. AJNR Am J Neuroradiol 1984;5:632-3.

17. Teplick JG, Haskin ME. Spontaneous regression of herniated nucleus pulposus. AJR American journal of roentgenology 1985;145:371-5.

18. Hirabayashi S, Kumano K, Tsuiki T, Eguchi M, Ikeda S. A dorsally displaced free fragment of lumbar disc herniation and its interesting histologic findings. A case report. Spine 1990;15:1231-

3.

19. Saal JA. Natural history and nonoperative treatment of lumbar disc herniation. Spine 1996;21:2S-9S.

20. Slavin KV, Raja A, Thornton J, Wagner FC, Jr. Spontaneous regression of a large lumbar disc herniation: report of an illustrative case. Surgical neurology 2001;56:333-6; discussion 7. 
21. Komori H, Shinomiya K, Nakai O, Yamaura I, Takeda S, Furuya K. The natural history of herniated nucleus pulposus with radiculopathy. Spine 1996;21:225-9.

22. Haro H, Shinomiya K, Murakami S, Spengler DM. Up-regulated expression of matrilysin and neutrophil collagenase in human herniated discs. Journal of spinal disorders 1999;12:245-9. 23. Henmi T, Sairyo K, Nakano S, et al. Natural history of extruded lumbar intervertebral disc herniation. J Med Invest 2002;49:40-3.

24. Urrutia J, Fadic R. Cervical disc herniation producing acute Brown-Sequard syndrome: dynamic changes documented by intraoperative neuromonitoring. European spine journal : official publication of the European Spine Society, the European Spinal Deformity Society, and the European Section of the Cervical Spine Research Society 2012;21 Suppl 4:S418-21.

25. Orief T, Orz Y, Attia W, Almusrea K. Spontaneous resorption of sequestrated intervertebral disc herniation. World Neurosurg 2012;77:146-52. 